\title{
Noninvasive assessment of cardiac changes in patients with coronavirus disease-19 (COVID-19) by bedside ultrasound
}

\author{
Ziqing Gao ${ }^{1}$, Yongquan Huang ${ }^{1}$, Wuzhu Lu ${ }^{1}$, Xiaobo Chen ${ }^{1}$, Xuefeng Li ${ }^{1}$, Shushan Zhang ${ }^{1}$, Zhongzhen Su ${ }^{1,2,3}$ \\ ${ }^{1}$ Department of Ultrasound, The Fifth Affiliated Hospital of Sun Yat-sen University, Zhuhai, China; ${ }^{2}$ Interventional Medicine, The Fifth Affiliated \\ Hospital of Sun Yat-sen University, Zhuhai, China; ${ }^{3}$ Guangdong Provincial Key Laboratory of Biomedical Imaging, The Fifth Affiliated Hospital of \\ Sun Yat-sen University, Zhuhai, China \\ Contributions: (I) Conception and design: Z Gao; (II) Administrative support: Z Su; (III) Provision of study materials or patients: Y Huang, W Lu; (IV) \\ Collection and assembly of data: Z Gao; (V) Data analysis and interpretation: Z Gao; (VI) Manuscript writing: All authors; (VII) Final approval of \\ manuscript: All authors. \\ Correspondence to: Zhongzhen Su, MD. Professor, Department of Ultrasound, The Fifth Affiliated Hospital of Sun Yat-sen University, Meihua Dong \\ Road, No. 52, Zhuhai 519000, China. Email: suzhzh3@mail.sysu.edu.cn.
}

Background: Patients with coronavirus disease-19 (COVID-19) are susceptible to cardiac complications, and cardiac ultrasound is the preferred noninvasive method for clinical cardiac structure and function assessment.

Methods: We performed a retrospective study of 34 patients with COVID-19 and analyzed their clinical data, biochemistry test results [creatine kinase-MB (CK-MB), cardiac troponin I (cTnI) and C-reactive protein (CRP)], and parameters of cardiac ultrasound [left atrium (LA), left ventricular end-diastolic dimensions (LVDD), right atrium (RA), right ventricle (RV), main pulmonary artery (MPA), left ventricular ejection fraction (LVEF), tricuspid valve (TV), pulmonic valve (PV) and pulmonary artery systolic pressure (PASP)]. We classified the patients based on their clinical symptoms (mild, moderate, severe, and critical groups), and compared the parameters. CK-MB and cardiac ultrasound parameters are presented as mean \pm standard deviation and compared using the one-way ANOVA. CTnI is presented as counts (percentages) and compared using the $\chi^{2}$ test, CRP is presented as $\left[\mathrm{M}\left(\mathrm{P}_{25}, \mathrm{P}_{75}\right)\right]$ [median (interquartile range)] and compared using nonparametric tests (Kruskal-Wallis test).

Results: As the disease progressed, the parameters of both biochemical blood tests and cardiac ultrasound changed regularly, manifested as enlargement of the LA, LVDD, RA, RV, and MPA and increased PASP, CRP, CK-MB, and cTnI. Of these parameters, CRP, LA, LVDD, MPA, and PASP of the severe group were more notably elevated than in the mild and moderate groups $(\mathrm{P}<0.05)$. The critical group more showed markedly increased CK-MB, cTnI, and RA than the other groups $(\mathrm{P}<0.05)$, and CRP, LA, LVDD, RV, MPA, and PASP rose more sharply than in the mild and moderate groups $(\mathrm{P}<0.05)$.

Conclusions: As COVID-19 progressed, patients developed an enlarged heart with expanded pulmonary arteries and elevated PASP. Bedside ultrasound can be used as a noninvasive assessment of these changes and for guidance of clinical treatment.

Keywords: Coronavirus disease-19 (COVID-19); cardiac ultrasound; myocardial damage

Submitted Nov 30, 2021. Accepted for publication Jan 04, 2022.

doi: $10.21037 /$ jtd-21-1961

View this article at: https://dx.doi.org/10.21037/jtd-21-1961

(c) Journal of Thoracic Disease. All rights reserved. 


\section{Introduction}

Since its outbreak in December 2019, coronavirus disease 2019 (COVID-19) has quickly spread through over 200 countries and regions worldwide and has become a public health emergency of international concern. The pathogen of COVID-19 is a novel coronavirus named severe acute respiratory syndrome coronavirus 2 (SARS-CoV-2), which is highly pathogenic and contagious. Clinical characteristics of SARS-CoV-2 infection typically include fever and respiratory symptoms. Furthermore, a significant proportion of patients present with cardiac symptoms. According to recent reports, cardiac complications are not only common (20-25\%) in COVID-19 infection, but they are also related to increased morbidity (1), such as arrhythmia (16.7\%) and acute myocardial damage (7.2\%), and death can occur in severe cases (10.5\%) (2). COVID-19 may aggravate pre-existing cardiovascular disease and trigger new and more serious heart damage, which may cause heart failure, cardiogenic shock, and even death in severe and critical cases (3). However, systematic cardiac imaging has not been described in these reports, and it has not been reported that cardiac changes can be assessed by cardiac ultrasound combined with myocardial enzymes. As the mainstay of cardiac imaging, echocardiography is used for the diagnosis of different causes of heart failure (4). Together with myocardial enzymes and other biochemical blood parameters, in this study we aimed to summarize the cardiac ultrasound characteristics of patients with COVID-19 and explore the clinical value of assessment of cardiac damage in patients with COVID-19. We present the following article in accordance with the STROBE reporting checklist (available at https://jtd. amegroups.com/article/view/10.21037/jtd-21-1961/rc).

\section{Methods}

The institutional review board approved this retrospective study that evaluated de-identified data and involved no potential risk to patients (ethical code: 2020, L033-1). Written informed consent was waived. No link between the patients and the researchers was made available to avert any potential breach of confidentiality. This study was conducted in accordance with the current version of the Declaration of Helsinki (as revised in 2013).

\section{Participants}

In this study, patients with COVID-19 admitted to the
Fifth Affiliated Hospital of Sun Yat-sen University from January 20 to April 10, 2020, were selected as research subjects. Based on their clinical symptoms, all the selected patients were classified as mild (with mild symptoms and no signs of pneumonia on imaging), moderate (showing fever and respiratory symptoms with radiological findings of pneumonia), severe (adult cases meeting any of the following criteria: (I) respiratory distress ( $\geq 30$ breaths/min); (II) pulse oxygen saturation $\leq 93 \%$ in the resting state; (III) alveolar oxygen partial pressure $\left(\mathrm{PaO}_{2}\right) /$ fraction of inspired oxygen $\left(\mathrm{FiO}_{2}\right) \leq 300 \mathrm{mmHg}(1 \mathrm{mmHg}=0.133 \mathrm{kPa})$. Patients whose chest imaging show obvious lesion progression $>50 \%$ within 24-48 h were managed as severe cases), and critical cases (meeting any of the following criteria: (I) respiratory failure and requiring mechanical ventilation; (II) shock; (III) with other organ failure that required intensive care unit care). After confirmation of COVID-19 and clinical classification, patients underwent biochemical blood tests and bedside echocardiography immediately. Inclusion criteria were as follows: patients with positive results for the COVID-19 nucleic acid tests (RT-PCR method), in accordance with definitions of confirmed cases in Diagnosis and Treatment Protocol for Novel Coronavirus Pneumonia (Trial Version 7) (3) released by the National Health Commission of the People's Republic of China. None of the patients had the delta virus. Exclusion criteria were: (I) patients who lacked biochemical blood tests or bedside cardiac ultrasound examination; (II) patients whose bedside echocardiography images were of poor quality and could not be used for image analysis; (III) patients who suffered an acute myocardial infarction, rheumatic heart disease (acute), or other cardiac diseases, or underwent cardiac surgery within 1 month; (IV) patients with chronic obstructive pulmonary disease, cor pulmonale, or other pulmonary diseases.

\section{Study methods}

Biochemical blood test: The levels of serum markers of myocardial damage and inflammation were measured for all the patients, including creatine kinase-MB (CK-MB), cardiac troponin I (cTnI), and C-reactive protein (CRP).

Echocardiography: A Philips Affinity 50 Color Doppler Diagnostic Ultrasound System (Philips, Bothell, WA, USA) equipped with an S4-2 phased array transducer (bandwidth: 2-4 MHz) was used. The examinations at the bedside were all performed by the same ultrasound physician with $>10$ years of clinical ultrasound experience. While in a resting state the patient was placed in the left lateral 
decubitus and supine positions and observed mainly in the parasternal long-axis, aorta, and apical four-chamber views. The M-mode and two-dimensional images were recorded to measure the dimensions of the left atrium (LA), right atrium (RA), right ventricle (RV), and main pulmonary artery (MPA), as well as the left ventricular end-diastolic dimensions (LVDD). The left ventricular ejection fraction (LVEF) was measured by M-mode ultrasound. The velocity of the tricuspid valve (TV) and pulmonary valve (PV), tricuspid regurgitation (TR) velocity $\left(\mathrm{TR}_{\max }\right)$, and other data were measured by the continuous wave Doppler ultrasound. Pulmonary artery systolic pressure (PASP) was calculated from the $\mathrm{TR}$ velocity $\left(\mathrm{TR}_{\max }\right)$, and the formula was $\mathrm{PASP}=4$ $\left(\mathrm{TR}_{\max }\right)^{2}+$ right atrial pressure. The diagnostic criterion for pulmonary hypertension was PASP $\geq 35 \mathrm{mmHg}$ (4). The criteria for abnormal ultrasound findings were based on the Consensus for Standard Assessment by Echocardiography in Chinese Adults with Heart Failure (5).

\section{Statistical analysis}

The statistical analysis of this study was performed using SPSS (version 24; IBM, New York, NY, USA). Enumeration data are presented as counts (percentages), and differences among groups were compared using the $\chi^{2}$ test. Measurement data are presented as mean \pm standard deviation and compared using the one-way ANOVA. [M $\left(\mathrm{P}_{25}, \mathrm{P}_{75}\right)$ ] [median (interquartile range)] was given and compared using nonparametric tests (Kruskal-Wallis test) when the data were not normally distributed. $\mathrm{P}<0.05$ was considered statistically significant.

\section{Results}

\section{Clinical features}

A total of 34 patients with COVID-19 (25 male and 9 female patients, age range: $18-80$ years) were included in this study. The procedure for selection is shown in Figure 1. Patients in the mild group were significantly younger than those in the other three groups $(\mathrm{P}<0.05)$. There was no statistical difference in underlying diseases among the patients with COVID-19 (P>0.05). The clinical characteristics are shown in Table 1.

\section{Parameters of cardiac enzymes (CK-MB and cTnI) and CRP}

The critical group showed a significant increase in the level of CK-MB and cTnI compared with the other three groups $(\mathrm{P}<0.05)$, among which there were no statistical differences $(\mathrm{P}>0.05)$. The severe and critical groups showed a significant elevation in the level of CRP compared with the mild and moderate groups, as shown in Table 2.

\section{Cardiac ultrasound findings}

The severe and critical groups showed an increase in LA, LVDD, MPA, and PASP compared with the mild and moderate groups $(\mathrm{P}<0.05)$. The critical group showed a marked enlargement in RA compared with the other three groups $(\mathrm{P}<0.05)$. The critical group showed an increase in $\mathrm{RV}$ compared with the mild and moderate groups, which demonstrated statistically significant differences $(\mathrm{P}<0.05)$, as shown in Table 3 and Figure 2.

\section{Discussion}

COVID-19 can not only cause severe damage to the lungs as the target organs, but also damage to multiple organs $(2,3)$, including the heart, liver, kidney, and so on. Especially in critically ill patients, COVID-19 with comorbid cardiovascular disease is not clinically rare (6-8). Left or right ventricular abnormalities have been found in half of all patients with COVID-19 who underwent echocardiography, and those abnormalities are severe in 1 in 7 patients, according to data from 1,216 patients evaluated in 69 countries spanning six continents (9). COVID-19 may aggravate pre-existing cardiovascular disease and trigger new and more serious heart damage, which may cause heart failure, cardiogenic shock, and even death in severe and critical cases (3). Therefore, such indicators, including cardiac morphology and function as well as myocardial enzymes, are closely monitored clinically and timely treatment is also given according to their changes to reduce myocardial damage, lower the mortality rate and improve prognosis.

Given that CK-MB and cTnI exhibit a relatively strong sensitivity and specificity in the diagnosis of myocardial damage (10), the results of this study that CK-MB and cTnI in the critical group were significantly higher than those in the other groups $(\mathrm{P}<0.05)$ indicate that patients in the critical group had the most significant myocardial damage. This may be caused by the fact that in critically ill patients, abnormally elevated levels of inflammatory cytokines and overactivated immune cells enter the bloodstream and invade the vascular endothelial cells of the 


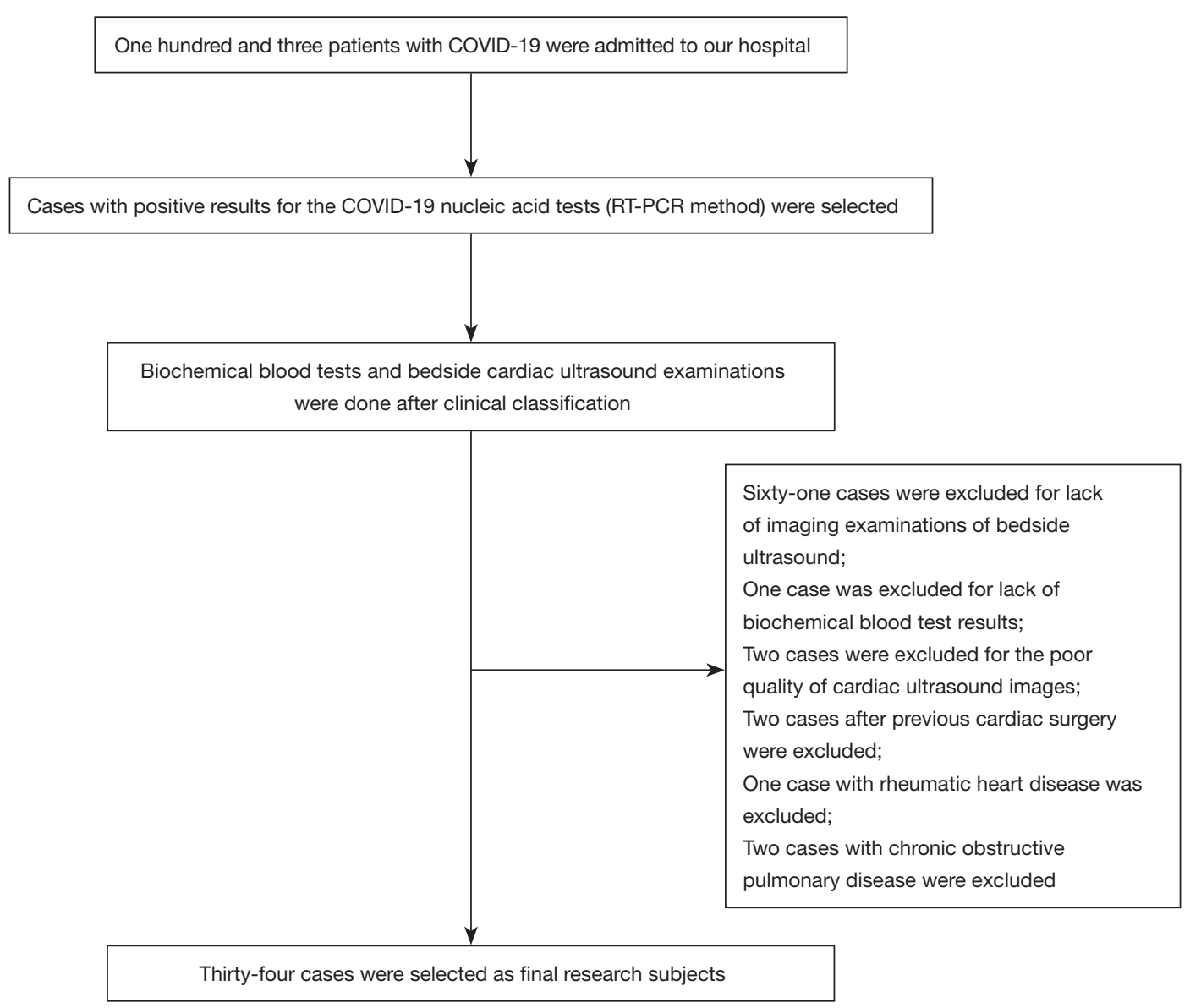

Figure 1 Flow chart of study inclusion. COVID-19, coronavirus disease-19.

heart, resulting in damage to vascular endothelial cells and increased vascular permeability, thus making it possible for inflammatory cells to penetrate the endodermis, infiltrate the myocardial tissue and directly lead to damage of myocardial cells. Meanwhile, endothelial cell damage causes vascular diastolic dysfunction and decreased coronary flow reserve, limiting the myocardial blood supply under the high load and thus bringing about myocardial ischemia (11), which further aggravates the myocardial damage. In this situation, CK-MB and cTnI significantly increase, with the end result of secondary cardiac enlargement in critically ill patients. However, it is still unclear whether SARSCoV-2 directly attacks the myocardium. According to the autopsy results reported by the team at the China Scientific Academy (12), the pathological changes in the lungs of the deceased patients were obvious, but no significant histological changes were observed in heart tissues, which indicates that SARS-CoV-2 may not directly damage the heart.
In addition, the results of this study showed that as disease severity progresses, the RA, RV, and MPA of the patients with COVID-19 tend to expand, and their PASP increases gradually. This may be caused by the large amounts of cytokines circulating in patients with COVID-19, which creates an "inflammatory cytokine storm" (13), inducing inflammatory response edema in the bronchi and alveoli, as well as inflammatory exudation, resulting in obstruction of ventilation and gas exchange, ventilation-perfusion imbalance, hypoxemia, carbon dioxide retention, and other symptoms. These responses reflexively provoke vasoconstriction of pulmonary small vessels and increase resistance. In addition, the hypoxia in the lung tissue and acidosis induced by the infection causes increased synthesis and secretion of prostaglandins, which further aggravates pulmonary small vessel contraction and spasm. The persistence of these changes leads to long-term contraction of pulmonary arterioles, which progresses with the severity of the disease, and ultimately leads to increased 
Table 1 Clinical characteristics of patients with COVID-19

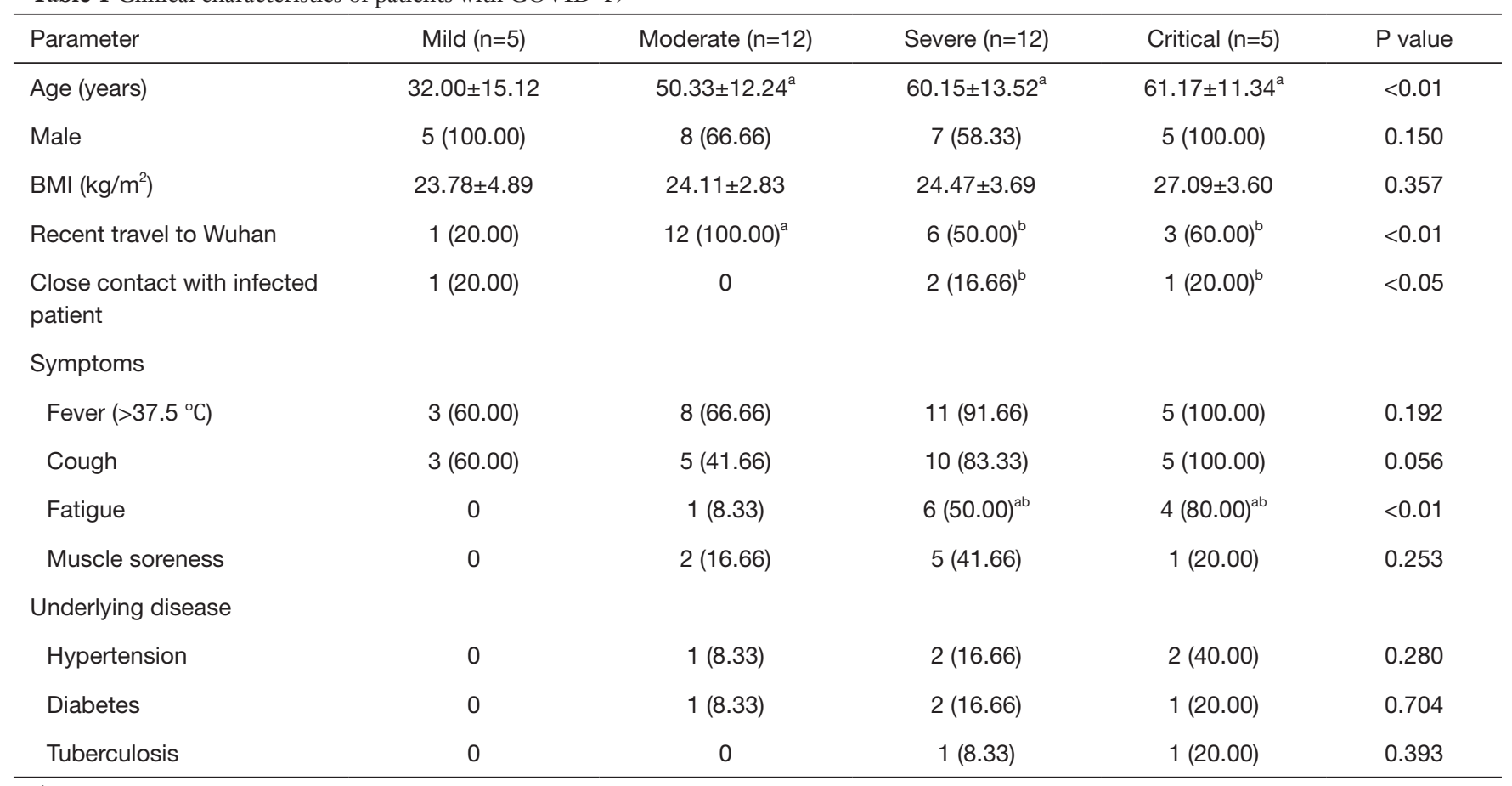

a,b , significant difference $(P<0.05)$ compared with the mild and moderate group, respectively. COVID-19, coronavirus disease-19.

Table 2 Comparison of CK-MB, cTnI and CRP in patients with COVID-19

\begin{tabular}{|c|c|c|c|c|c|}
\hline Parameter & Mild $(n=5)$ & Moderate $(n=12)$ & Severe $(n=12)$ & Critical $(n=5)$ & $P$ value \\
\hline cTnl & 0 & 0 & $1(8.33)$ & $5(100.00)^{\mathrm{abc}}$ & $<0.01$ \\
\hline $\operatorname{CRP}\left(\mathrm{M}\left(\mathrm{P}_{25}, \mathrm{P}_{75}\right) \mathrm{mg} / \mathrm{L}\right)$ & $3.36(0.71,16.93)$ & $1.38(0.55,16.10)$ & $29.52(10.31,74.33)^{\mathrm{ab}}$ & $58.01(34.51,85.36)^{\mathrm{ab}}$ & $<0.01$ \\
\hline
\end{tabular}

a,b,c, significant difference $(\mathrm{P}<0.05)$ compared with the mild, moderate and severe group, respectively. Abnormal levels of cTnl are presented as counts (percentages). CK-MB, creatine kinase-MB. cTnl, cardiac troponin I. CRP, C-reactive protein; COVID, coronavirus disease.

pulmonary arterial pressure (14) as well as pulmonary artery dilatation. Persistent pulmonary hypertension also further worsens the right heart enlargement. Clinical deterioration occurred in $20 \%$ of patients during hospitalization. Repeat echocardiography will reveal further deterioration of the RV parameters in those patients, probably related to elevated pulmonary pressure (15).

As for the increase in the CK-MB and cTnI levels in critically ill patients, we believe it can be attributed to the following. On the one hand, the infiltration of inflammatory cytokines and immune cells after pulmonary infection causes damage to myocardial cells. On the other hand, the increased pulmonary arterial pressure leads to increased right heart afterload and aggravated myocardial oxygen consumption. Given that the myocardium is extremely sensitive to hypoxia, this can directly lead to myocardial energy metabolism disorders, and further aggravate myocardial damage. Therefore, the prognosis of myocardial damage may depend more on the control of pulmonary inflammation and the degree of decrease in pulmonary arterial pressure. According to this inference, active treatment of pulmonary infection and monitoring of pulmonary arterial pressure may play an important role in promoting a good outcome of myocardial damage.

Cardiac ultrasound is the preferred method for clinical assessment of cardiac structure and function and for pulmonary arterial pressure monitoring. Our previous study showed that performing bedside ultrasound has 
Table 3 Results of cardiac ultrasound in patients with COVID-19

\begin{tabular}{|c|c|c|c|c|c|}
\hline Parameter & Mild $(n=5)$ & Moderate $(n=12)$ & Severe $(n=12)$ & Critical $(n=5)$ & $P$ value \\
\hline LVDD (mm) & $43.66 \pm 4.22$ & $44.50 \pm 3.26$ & $48.53 \pm 3.77^{\mathrm{ab}}$ & $52.00 \pm 7.37^{\mathrm{ab}}$ & $<0.01$ \\
\hline $\mathrm{RA}(\mathrm{mm})$ & $32.66 \pm 2.33$ & $34.58 \pm 1.24$ & $35.92 \pm 1.55^{\mathrm{a}}$ & $38.16 \pm 4.21^{\mathrm{abc}}$ & $<0.01$ \\
\hline $\mathrm{RV}(\mathrm{mm})$ & $32.83 \pm 3.31$ & $33.83 \pm 1.19$ & $35.15 \pm 1.90$ & $36.66 \pm 3.82^{\mathrm{ab}}$ & $<0.05$ \\
\hline LVEF (\%) & $67.33 \pm 5.16$ & $67.41 \pm 5.53$ & $66.61 \pm 3.57$ & $65.00 \pm 3.74$ & 0.745 \\
\hline $\mathrm{TV}(\mathrm{m} / \mathrm{s})$ & $0.60 \pm 0.12$ & $0.62 \pm 0.12$ & $0.65 \pm 0.15$ & $0.62 \pm 0.13$ & 0.882 \\
\hline $\mathrm{PV}(\mathrm{m} / \mathrm{s})$ & $0.88 \pm 0.15$ & $0.91 \pm 0.13$ & $0.87 \pm 0.13$ & $0.91 \pm 0.68$ & 0.848 \\
\hline PASP $(\mathrm{mmHg})$ & $26.00 \pm 2.82$ & $26.50 \pm 4.42$ & $36.60 \pm 7.55^{\mathrm{ab}}$ & $39.50 \pm 9.60^{\mathrm{ab}}$ & $<0.01$ \\
\hline
\end{tabular}

${ }^{a, b, c}$, significant difference $(P<0.05)$ compared with the mild, moderate and severe group, respectively. COVID, coronavirus disease; LA, left atrium. RA, right atrium. RV, right ventricle. MPA, main pulmonary artery. LVDD, left ventricular end-diastolic dimensions. LVEF, left ventricular ejection fraction. TV, velocity of the tricuspid valve. PV, pulmonary valve. PASP, Pulmonary artery systolic pressure.

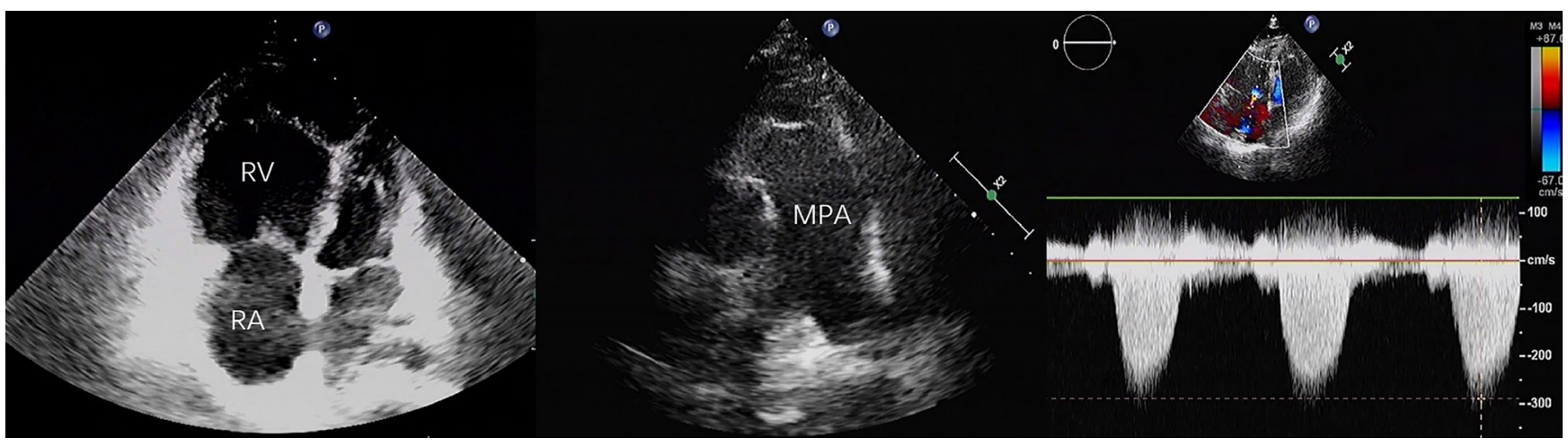

Figure 2 Cardiac ultrasound findings. The most critical patients showed an increase in RA, RV, MPA and PASP. RA, right atrium; RV, right ventricle; MPA, main pulmonary artery; PASP, pulmonary artery systolic pressure.

important clinical significance in the assessment and dynamic observation of pulmonary lesions in patients with COVID-19 (16). In one-third of patients who underwent echocardiography for clinical features, there was an instant change in patient management (17), such as diseasespecific therapies (e.g., pericardiocentesis or treatment for heart failure, pulmonary embolism, or acute coronary syndrome). The imaging results also influenced decisions regarding the level of care given to the patient, such as admission to intensive care and the need for hemodynamic support to be titrated (9). Abnormal cardiac structure was seen in approximately two-thirds of the patients with COVID-19 with myocardial injury who had undergone echocardiography. Myocardial injury was linked to increase in-hospital deaths, especially when echocardiographic abnormalities were present (18). Therefore, for infected patients with myocardial damage, bedside ultrasound can be used for combined monitoring of heart and lungs specifically, and dynamic monitoring of changes in condition, providing a reference for clinical treatment. Moreover, critically ill patients with COVID-19 often have multiple organ involvement, and bedside ultrasound facilitates immediate systemic assessment of the patient.

This study has several limitations. First, the sample size was small, so possible confounding factors that affect the results may occur, and thus expansion of the sample size and a multicenter study are needed. Second, this study was a retrospective analysis, lacking indicators related to the evaluation of right ventricular systolic and diastolic function, and no evaluation of right heart function. Third, we did not 
perform dynamic cardiac ultrasound monitoring in patients with COVID-19. Therefore, follow-up examinations are required to illustrate the ultrasound manifestations over the whole course of COVID-19.

In summary, patients with COVID-19 showed gradual enlargement of the heart and pulmonary arteries and elevated PASP as the disease progressed. Bedside ultrasound can be used for a noninvasive assessment of these changes, which may be helpful as a clinical guide to treatment.

\section{Acknowledgments}

Funding: This work was supported by the Natural Science Foundation of Guangdong Province (No. 2018A0303130070), the Science and Technology Projects of Zhuhai (No. ZH22036201210048PWC) and the Scientific Research Project of Traditional Chinese Medicine Bureau of Guangdong Province (No. 20201071).

\section{Footnote}

Reporting Checklist: The authors have completed the STROBE reporting checklist. Available at https://jtd. amegroups.com/article/view/10.21037/jtd-21-1961/rc

Data Sharing Statement: Available at https://jtd.amegroups. com/article/view/10.21037/jtd-21-1961/dss

Conflicts of Interest: All authors have completed the ICMJE uniform disclosure form (available at https://jtd.amegroups. com/article/view/10.21037/jtd-21-1961/coif). The authors have no conflicts of interest to declare.

Ethical Statement: The authors are accountable for all aspects of the work in ensuring that questions related to the accuracy or integrity of any part of the work are appropriately investigated and resolved. The institutional review board approved this retrospective study that evaluated de-identified data and involved no potential risk to patients (ethical code:2020, L033-1). Written informed consent was waived. No link between the patients and the researchers was made available to avert any potential breach of confidentiality. This study was conducted in accordance with the current version of the Declaration of Helsinki (as revised in 2013).

Open Access Statement: This is an Open Access article distributed in accordance with the Creative Commons
Attribution-NonCommercial-NoDerivs 4.0 International License (CC BY-NC-ND 4.0), which permits the noncommercial replication and distribution of the article with the strict proviso that no changes or edits are made and the original work is properly cited (including links to both the formal publication through the relevant DOI and the license). See: https://creativecommons.org/licenses/by-nc-nd/4.0/.

\section{References}

1. Zhou F, Yu T, Du R, et al. Clinical course and risk factors for mortality of adult inpatients with COVID-19 in Wuhan, China: a retrospective cohort study. Lancet 2020;395:1054-62.

2. Wang D, Hu B, Hu C, et al. Clinical Characteristics of 138 Hospitalized Patients With 2019 Novel CoronavirusInfected Pneumonia in Wuhan, China. JAMA 2020;323:1061-9.

3. National Health Commission of the People's Republic of China. Diagnosis and Treatment Protocol for Novel Coronavirus Pneumonia (trial version 7). Infect Dis Info 2020;133:1-6.

4. Guo T, Fan Y, Chen M, et al. Cardiovascular Implications of Fatal Outcomes of Patients With Coronavirus Disease 2019 (COVID-19). JAMA Cardiol 2020;5:811-8.

5. National Center for Gerontology National Clinical Research Center for Geriatric Disorders, Cardiovascular Branch of Chinese Geriatrics Society, Beijing Medical Association Imaging Group of Cardiovascular Department, et al. Consensus for Standard Assessment by Echocardiography in Chinese Adults with Heart Failure. Chinese Circulation Journal 2019;34:422-436.

6. Chen N, Zhou M, Dong X, et al. Epidemiological and clinical characteristics of 99 cases of 2019 novel coronavirus pneumonia in Wuhan, China: a descriptive study. Lancet 2020;395:507-13.

7. Huang C, Wang Y, Li X, et al. Clinical features of patients infected with 2019 novel coronavirus in Wuhan, China. Lancet 2020;395:497-506.

8. Guo D, Guo WG, Liu PY, et al., Cardiovascular Manifestations of 2019-nCoV Infection. Chin Heart J 2020;32:75-7.

9. Dweck MR, Bularga A, Hahn RT, et al. Global evaluation of echocardiography in patients with COVID-19. Eur Heart J Cardiovasc Imaging 2020;21:949-58.

10. Thygesen K, Alpert JS, Jaffe AS, et al. Fourth Universal Definition of Myocardial Infarction. Circulation 2018;138:e618-e651. 
11. Brutsaert DL, Housmans PR, Goethals MA. Dual control of relaxation. Its role in the ventricular function in the mammalian heart. Circ Res 1980;47:637-52.

12. Xu Z, Shi L, Wang Y, et al. Pathological findings of COVID-19 associated with acute respiratory distress syndrome. Lancet Respir Med 2020;8:420-2.

13. Liu J, Zheng X, Tong Q, et al. Overlapping and discrete aspects of the pathology and pathogenesis of the emerging human pathogenic coronaviruses SARS-CoV, MERS-CoV, and 2019-nCoV. J Med Virol 2020;92:491-4.

14. Verma B, Daga SR, Mahapankar A. Persistent pulmonary hypertension among neonates with sepsis. Indian J Pediatr 2006;73:250-1.

Cite this article as: Gao Z, Huang Y, Lu W, Chen X, Li X, Zhang S, Su Z. Noninvasive assessment of cardiac changes in patients with coronavirus disease-19 (COVID-19) by bedside ultrasound. J Thorac Dis 2022;14(2):423-430. doi: 10.21037/jtd21-1961
15. Szekely Y, Lichter Y, Taieb P, et al. Spectrum of Cardiac Manifestations in COVID-19: A Systematic Echocardiographic Study. Circulation 2020;142:342-53.

16. Lu W, Zhang S, Chen B, et al. A Clinical Study of Noninvasive Assessment of Lung Lesions in Patients with Coronavirus Disease-19 (COVID-19) by Bedside Ultrasound. Ultraschall Med 2020;41:300-7.

17. Lazzeri C, Bonizzoli M, Batacchi S, et al. The clinical and prognostic role of echocardiography in 'SARS-CoV-2' disease. Eur J Emerg Med 2021;28:321-3.

18. Giustino G, Croft LB, Stefanini GG, et al. Characterization of Myocardial Injury in Patients With COVID-19. J Am Coll Cardiol 2020;76:2043-55. 